\title{
Reeb Embeddings and Universality of Euler Flows
}

\author{
Robert Cardona, Eva Miranda, Daniel Peralta-Salas, and Francisco Presas
}

\begin{abstract}
We use a new geometrical approach to the universality of Euler flows. By proving flexibility results on embeddings for Reeb flows in contact topology, we deduce some new universal properties for Euler flows. As a byproduct, we deduce the Turing completeness of stationary Euler flows, answering an open question for steady solutions. The results contained in this article are an announcement and short version of [2], where the complete list of results and proofs can be found.
\end{abstract}

Robert Cardona acknowledges financial support from the Spanish Ministry of Economy and Competitiveness, through the María de Maeztu Programme for Units of Excellence in R\&D (MDM-20140445). Robert Cardona and Eva Miranda are supported by the grants MTM2015-69135-P/FEDER and PID2019-103849GB-I00/AEI/10.13039/501100011033, and AGAUR grant 2017SGR932. Eva Miranda is supported by the Catalan Institution for Research and Advanced Studies via an ICREA Academia Prize 2016. Daniel Peralta-Salas is supported by the grants MTM PID2019-106715GBC21 (MICINN) and Europa Excelencia EUR2019-103821 (MCIU). Francisco Presas is supported by the grant reference number MTM2016-79400-P (MINECO/FEDER). This work was partially supported by the ICMAT-Severo Ochoa grant SEV-2015-0554.

R. Cardona $(\varangle) \cdot$ E. Miranda

Laboratory of Geometry and Dynamical Systems, Department of Mathematics EPSEB,

Universitat Politècnica de Catalunya BGSMath Barcelona Graduate School of Mathematics in

Barcelona, Barcelona, Spain

e-mail: robert.cardona@upc.edu

E. Miranda

e-mail: eva.miranda@upc.edu

E. Miranda

IMCCE, CNRS-UMR8028, Observatoire de Paris, PSL University, Sorbonne, Université, 77

Avenue Denfert-Rochereau, 75014 Paris, France

D. Peralta-Salas $\cdot$ F. Presas

Instituto de Ciencias Matemàticas ICMAT-CSIC, Madrid, Spain

e-mail: dperalta@icmat.es

F. Presas

e-mail: fpresas@icmat.es

(C) The Author(s), under exclusive license to Springer Nature Switzerland AG 2021

M. Alberich-Carramiñana et al. (eds.), Extended Abstracts GEOMVAP 2019,

Trends in Mathematics 15,

https://doi.org/10.1007/978-3-030-84800-2_19 


\section{Introduction}

\subsection{Hydrodynamics}

The Euler equations model ideal fluids, and can be formulated on a Riemannian manifold $(M, g)$ as follows,

$$
\left\{\begin{array}{ll}
\partial_{t} u+\nabla_{u} u & =-\operatorname{grad}_{g} p \\
\operatorname{div}_{g} u & =0
\end{array},\right.
$$

where $p$ is the pressure function and $u$ the velocity field. In the articles $[9,10]$ Tao anticipated that Euler flows should be flexible enough to show any kind of dynamical behavior. Instead of using an analytical approach we will use a geometrical one to prove universality properties. In particular, in [10] the extendibility of flows to Euler solutions is studied, and existence of Turing complete Euler flows is left open. Turing completeness of Euler flows means that there is a solution to the Euler equations associated to a general Riemannian manifold encoding a universal Turing machine. We will address this question in the stationary case.

If we restrict ourselves to autonomous solutions to the Euler equations in odd dimensional manifolds, a rich geometric and topological study has been developed since the monograph [1]. Denoting $\alpha=\iota_{u} g$ the dual form to the velocity field, the stationary Euler equations can be written,

$$
\left\{\begin{array}{l}
\iota_{u} d \alpha=-d B \\
d \iota_{u} \mu=0,
\end{array}\right.
$$

where $B=P+\iota_{u} \alpha$ is the Bernoulli function and $\mu$ is the Riemannian volume form. This formulation establishes a dichotomy. When the Bernoulli function is non-constant (and for instance analytical or $C^{2}$ and Morse-Bott) then by Arnold's structural theorem the flow lines have a very rigid structure and dynamics are similar to those exhibited in integrable systems. However, when $B$ is constant the dynamics can be much more complicated. These solutions satisfy that the velocity field is parallel to the vorticity field $\omega$ defined by the equation

$$
\iota_{\omega} \mu=(d \alpha)^{n}
$$

We call a vector field $X$ a Beltrami field if it preserves $\mu$ and is everywhere parallel to its vorticity field $\omega$. We call it rotational if the function $\lambda \in C^{\infty}(M)$ such that $\omega=\lambda X$ satisfies $\lambda \neq 0$. 


\subsection{Contact Geometry}

At this point contact geometry appears as one of the interesting geometrical structures underlying the Euler equations. Let us recall some basic properties of contact structures.

Definition 1 Let $M^{2 n+1}$ be an odd dimensional manifold equipped with a hyperplane distribution $\xi$ such that there is a 1-form $\alpha \in \Omega^{1}(M)$ with $\operatorname{ker} \alpha=\xi$ and $\alpha \wedge(d \alpha)^{n} \neq$ 0 everywhere. Then $\left(M^{2 n+1}, \xi\right)$ is a (cooriented) contact manifold.

The contact structure $\xi$ does not depend on the choice of the defining one form, called the contact form. There is a unique vector field $R$ for a given $\alpha$ defined by the equations

$$
\left\{\begin{array}{l}
\iota_{R} \alpha=1 \\
\iota_{R} d \alpha=0
\end{array}\right.
$$

called the Reeb vector field.

The relation of Reeb vector fields and the Euler equations is provided by the following theorem by Etnyre and Ghrist [4].

Theorem 2 Any nonsingular rotational Beltrami field is a reparametrization of a Reeb vector field defined by some contact form. Any reparametrization of a Reeb vector field defined by a contact form is a nonsingular rotational Beltrami field for some metric and volume form.

This theorem allows us to introduce the techniques used in contact geometry in the study of some steady Euler flows, for instance the flexibility shown through the $h$-principle techniques introduced by Gromov [5] (see [3] for a modern text and extension of these results).

\section{Reeb Embeddings}

In order to find new universality properties for Euler flows, we adapt the questions of Tao to this setting. We ask the following question: given an arbitrary non-vanishing vector field $X$ on a closed manifold $N$, can we "embed" it in a Reeb flow of a bigger manifold for instance the standard contact sphere? More precisely, can we find a contact form $\alpha$ with Reeb vector field $R$ in a bigger closed manifold $M$ such that it has an invariant submanifold diffeomorphic to $N$ with $\left.R\right|_{N}=X$ ?

As we will see in a moment, $X$ needs to satisfy at least one extra condition. Let us recall the following definition.

Definition 3 A vector field $X$ in a manifold $M$ is called geodesible if its orbits are geodesics for some metric in $M$. 
We will assume from now on that $X$ is geodesible of unit-length (i.e. it is of unitlength for the metric making its orbits geodesics). It is well known following works of Gluck [6] that an equivalent condition for $X$ to be geodesible is that there exists a one form $\beta$ such that

$$
\iota_{X} \beta=1 \text {, and } \iota_{X} d \beta=0
$$

It becomes now clear that a Reeb vector field $R$ is geodesible: the contact form $\alpha$ satisfies the necessary conditions. Furthermore if $W$ is an invariant submanifold of $R$, then $R$ is also geodesible in $W$ since the restriction of the contact form to $W$ satisfies also Gluck's conditions. This implies that vector fields that can potentially be embedded as a Reeb field need to be geodesible. However, we prove that this is the only condition: any geodesible vector field can be embedded as a Reeb vector field of the same manifold: the standard contact sphere. This is contained in the following theorem, which is a weaker version of the main Theorem 5 that will be discussed below.

Theorem 4 ([2]) Let $N$ be a closed manifold of dimension $n$ and $X$ a geodesible flow on it, then there is an embedding of $N$ in $\left(S^{4 n-1}, \xi_{\text {std }}\right)$ and a form $\beta$ defining $\xi_{\text {std }}$ such that the Reeb field $R$ of $\beta$ satisfies $\left.R\right|_{N}=X$.

The proof of this theorem is less technical than the main theorem, let us give a sketch. The proofs with all the details can be found in [2].

Sketch of proof. We start with a geodesible vector field $X$ on a closed manifold $N$. This means that for some form $\alpha$ we have $\iota_{X} \alpha>0$ and $\iota_{X} d \alpha=0$. This implies that $X$ preserves $\eta=\operatorname{ker} \alpha$. In fact being geodesible is equivalent to saying that $X$ preserves a transversal hyperplane field.

(1) We begin constructing an open contact manifold $M$ containing $N$ with a symplectic hyperplane distribution, that contains $\eta$ when restricted to $N$. We consider the vector bundle $\pi: \eta^{*} \rightarrow N$ over $N$ with the hyperplane distribution $\tilde{\eta}=\pi^{*}\left(\eta \oplus \eta^{*}\right)$, which is equipped with the canonical symplectic structure.

(2) We now obtain a contact structure that also contains $\eta$ when restricted to $N$. To do this we perturb the symplectic form in an appropriate way and work chart by chart to find a contact structure $\bar{\alpha}$ satisfying ker $\left.\bar{\alpha}\right|_{N} \cap T N=\eta$

(3) We apply an $h$-principle result by Gromov on isocontact embeddings that tells us that $M$ embeds isocontactly in $\left(S^{4 n-1}, \xi_{s t d}\right)$. This means that there is an embedding $e: N \hookrightarrow S^{4 n-1}$ such that $e^{*} \xi_{s t d}=\operatorname{ker} \tilde{\alpha}$. We have that our vector field $X$ in $e(N)$ preserves $\xi_{s t d} \cap T N=\eta$ by construction. By a characterization of Inaba [7], this condition is equivalent to the existence of a contact form defining $\xi_{\text {std }}$ such that its Reeb vector field $R$ satisfies $\left.R\right|_{N}=X$.

This theorem is merely geometrical and allows the realization of any geodesible field on a manifold of dimension $n$ as the Reeb vector field in the standard contact sphere $S^{4 n-1}$. However, it is a particular instance of the following more general theorem. 
Theorem 5 ([2]) Let $e:(N, X) \hookrightarrow\left(M, \xi^{\prime}\right)$ be a smooth embedding of $N$ into a contact manifold $\left(M, \xi^{\prime}\right)$ where $X$ is a geodesible vector field on $N$. Assume that $\operatorname{dim} M \geq 3 n+2$. Then $e$ is isotopic to a Reeb embedding $\tilde{e}$, and $\tilde{e}$ can be taken $C^{0}$-close to $e$.

This result is stronger, and interesting from the contact topology perspective. It relies on defining a formal counterpart of a Reeb embedding and proving that it satisfies a certain $h$-principle. Then Theorem 5 follows from proving that smooth embeddings in high enough codimension are formal Reeb embeddings satisfying the $h$-principle. We will use this last Theorem to deduce the universality properties of Euler flows, since the resulting dimension of the ambient manifold is smaller.

\section{Applications}

Realizing geodesible vector fields as Reeb vector will implies different universality properties for Euler flows, and we state some of them here. Because of the correspondence of Reeb vector fields and Beltrami fields, we know already that every geodesible vector field can be embedded in a stationary solution to the Euler equations. The following corollaries are obtained by finding the appropriate geodesible vector field and applying Theorem 5 .

We define the suspension of a time-periodic vector field $X(t)$ on a manifold $N$ (of dimension $n$ ) as the manifold $N \times S^{1}$ endowed with the vector field $Y=$ $(X(p, \theta), \partial \theta)$. It is a geodesible field and hence

Corollary 6 ([2]) Let $X(t)$ any periodic on time vector field on $N$. Then its suspension $Y$ in $N \times S^{1}$ can be embedded as a Reeb flow on $S^{m}$ with $m$ the odd integer in $\{3 n+5,3 n+6\}$.

In particular this proves that any periodic in time vector field can be extended to an Euler solution in the sense of [10].

In a similar way, given an orientation preserving diffeomorphism, one can consider the manifold $\tilde{N}=N \times[0,1] / \sim$ identifying $(x, 0)$ with $(\varphi(x), 1)$. Consider the horizontal flow on it

$$
\phi_{t}(\theta, x)=(\theta+t, x)
$$

The vector field obtained by this flow is geodesible and has as return map the given diffeomorphism.

Corollary 7 ([2]) Let $\varphi: N \rightarrow N$ be an orientation preserving diffeomorphism. It can be realized as the return map on some cross section diffeomorphic to $N$ in some Reeb flow on $S^{m}$ with $m$ the odd integer in $\{3 n+5,3 n+6\}$.

The Reeb flow can always be obtained on the standard contact sphere $\left(S^{3 n+5}, \xi_{\text {std }}\right)$ or $\left(S^{3 n+6}, \xi_{s t d}\right)$ depending on the parity of $n$ (since the dimension of the sphere needs to be odd). 
As a byproduct, we obtain the existence of Turing complete Euler flows. This is a consequence of the fact that there exists an orientation-preserving diffeomorphism of $\mathbb{T}^{4}$ encoding a universal Turing machine, see [8].

Corollary 8 ([2]) There are Reeb flows (and hence Euler flows) which are Turing complete. Concretely, there is a Reeb flow on $\left(S^{17}, \xi_{\text {std }}\right)$ encoding a universal Turing machine.

In his papers, Tao speculates on using a Turing complete flow to construct a finite time blow up solution to the Euler or Navier-Stokes equations. We do not know how this solution evolves when taken as initial condition for the Navier-Stokes equation.

\section{References}

1. V.I. Arnold, B. Khesin, Topological Methods in Hydrodynamics (Springer, New York, 1999)

2. R. Cardona, E. Miranda, D. Peralta-Salas, F. Presas. Universality of Euler flows and flexibility of Reeb embeddings. Preprint (2019). arXiv:1911.01963

3. Y. Eliashberg, N. Mishachev, Introduction to the h-Principle, Graduate Studies in Mathematics, vol. 48. (American Mathematical Society, Providence, RI, 2002)

4. J. Etnyre, R. Ghrist, Contact topology and hydrodynamics I. Beltrami fields and the Seifert conjecture. Nonlinearity 13(2), 441-458 (2000)

5. M. Gromov, Partial Differential Relations. Ergebnisse der Mathematik und ihrer Grenzgebiete 9 (Springer, Berlin, 1986)

6. H. Gluck, Open letter on geodesible flows

7. T. Inaba, Extending a vector field on a submanifold to a Reeb vector field on the whole contact manifold. Unpublished paper (2019). http://www.math.s.chiba-u.ac.jp/ inaba/

8. T. Tao, On the universality of potential well dynamics. Dyn. PDE 14, 219-238 (2017)

9. T. Tao, On the universality of the incompressible Euler equation on compact manifolds. Discret. Contin. Dyn. Syst. A 38(3), 1553-1565 (2018)

10. T. Tao, On the universality of the incompressible Euler equation on compact manifolds, II. Nonrigidity of Euler flows. Pure Appl. Function. Anal. 5(6), 1425-1443 (2020). arXiv:1902.0631 